\title{
Research on Sales Level Forecast of New Energy Vehicles based on BP Neural Network Model
}

\author{
Lingling Lu*, Yaqi Zhang, Qianwei Zong, Lulu Xue
}

Anyang Normal University, Anyang, China, 455000

*Corresponding Author

Lingling Lu

\section{Article History}

Received: 05.11.2019

Accepted: 12.11.2019

Published: 15.11 .2019

\begin{abstract}
This paper first analyzes the development trend and regularity of the production and sales of new energy vehicles in the first half of 2017-2019, and then establishes a BP neural network model and predicts sales in the third and fourth quarters of 2019. Then it studies the development trend of new energy vehicles and puts forward some suggestions for promoting the development of new energy vehicles.
\end{abstract}

Keywords: New energy vehicle, sales forecast, BP neural network.

\section{INTRODUCTION}

New energy vehicle is one of the ten fields in which China implements the first ten-year action plan of manufacturing power strategy. The development of new energy vehicle is in line with the strategic thought of "promoting green development" in the 19th National Congress of the Communist Party of China (CPC). Under the guidance and support of the national policy, China's new energy automobile industry has developed rapidly, and the automobile industry has become the pillar of the national economy, which is of great significance to the development of the national economy.

According to the data, the annual output of new energy vehicles in 2017 was 794000 units, up 53.8 percent from the same period last year, and the sales volume was 777000 units, up 53.3 percent from the same period last year. In 2018, the annual output of new energy vehicles was 149000 vehicles, up 68.5 percent from the same period last year, and the sales volume was 163000 vehicles, up 56.8 percent from the same period last year. However, by September 2019, the production of new energy vehicles was 2.209 million, down 6.2 percent from the same period last year, and sales were 2.271 million, down 5.2 percent from the same period last year, although the decline in production narrowed, production and sales fell again after July and August. Based on the large-scale promotion of the purchase of new energy vehicles, the policy of eliminating the number of new energy vehicles is gradually unable to balance the advantages and disadvantages of new energy vehicles and fuel vehicles, so sales of new energy vehicles have become less and less from 2017 to 2019. However, the above data only analyze and study the new energy vehicles based on the existing data, and do not make a reasonable forecast of future car sales. According to the trend of decreasing automobile sales, the state and the government need to work out the corresponding compensation plan to meet the demand of the future market, so it is particularly important for the policy makers to accurately predict the development and growth trend of the automobile market, as well as the operators who study the market market to formulate marketing strategy, so it is necessary to deal with and forecast the sales volume of new energy vehicles. It is a hot topic to predict the development trend and sales volume of new energy vehicles in the future by using mathematical model method. In this paper, the production and sales data of new energy vehicles in China from 2017 to 2019 are analyzed, and then the BP neural network model is established to predict the sales in the third quarter and the fourth quarter of 2019. Then, according to the prediction results, this paper analyzes the future development trend of new energy vehicles, and finally puts forward some corresponding suggestions to optimize the allocation of resources and reduce the risk of decision-making.

\section{Analysis of Index Factors Affecting New Energy Vehicles}

Indicators affecting sales of new energy vehicles are weight, horsepower, mileage, consumer preferences, quarterly, etc. In order to facilitate the analysis, this paper uses the principal component analysis in SPSS software, and under the condition that the cumulative variance contribution rate is not less than $85 \%$, it is concluded that the top three indexes are vehicle weight, horsepower

Copyright @ 2019: This is an open-access article distributed under the terms of the Creative Commons Attribution license which permits unrestricted use, distribution, and reproduction in any medium for non commercial use (NonCommercial, or CC-BY-NC) provided the original author and source are credited. 
and endurance mileage, and their variance contribution rates are $67.58 \%, 14.21 \%$ and $8.9 \%$, respectively. Therefore, this paper selects car weight, horsepower and mileage as influence variables. This paper analyzes the impact of new energy vehicle sales based on Table-1 data (see Fig-1).

Table-1: Quarterly sales figures for 2016-2019

\begin{tabular}{|c|c|c|c|c|}
\hline Date & Horsepowe & Car weight & Continuous mileage & Real sales volume \\
\hline The first quarter of 2016 & 178.66 & 1786.4 & 62.5 & 5.8 \\
\hline The two quarter of 2016 & 182.48 & 1752.52 & 80.4 & 11.1 \\
\hline The three quarter of 2016 & 180.21 & 1745.84 & 91.94 & 12.2 \\
\hline The four quarter of 2016 & 167.41 & 1729.19 & 97.37 & 21.3 \\
\hline$\ldots$ & $\ldots$ & $\ldots$ & $\ldots$ & $\ldots$ \\
\hline The first quarter of 2019 & 165.26 & 1733.8 & 118.79 & 35.3 \\
\hline The two quarter of 2019 & 165.26 & 1729.8 & 126.79 & \\
\hline The three quarter of 2019 & 165.28 & 1733.8 & 117.79 & \\
\hline The four quarter of 2019 & 165.2 & 1731.8 & 122.79 & \\
\hline
\end{tabular}

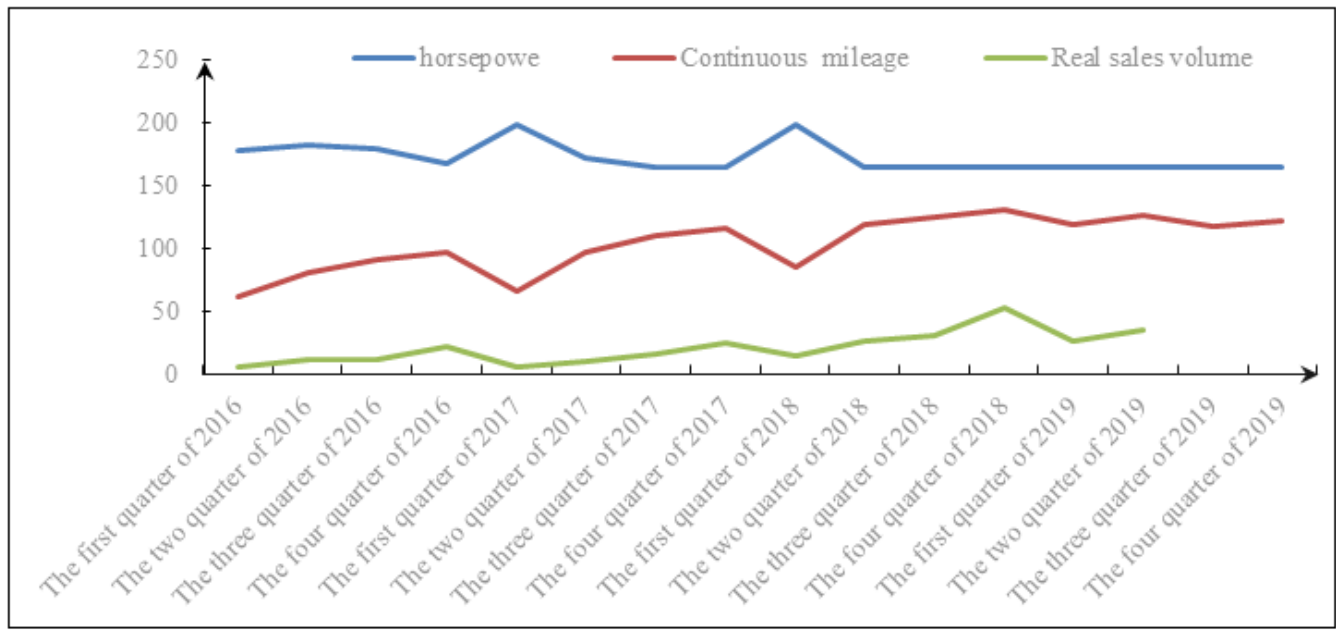

Fig-1: A broken line diagram of the relationship between horsepower and mileage and sales

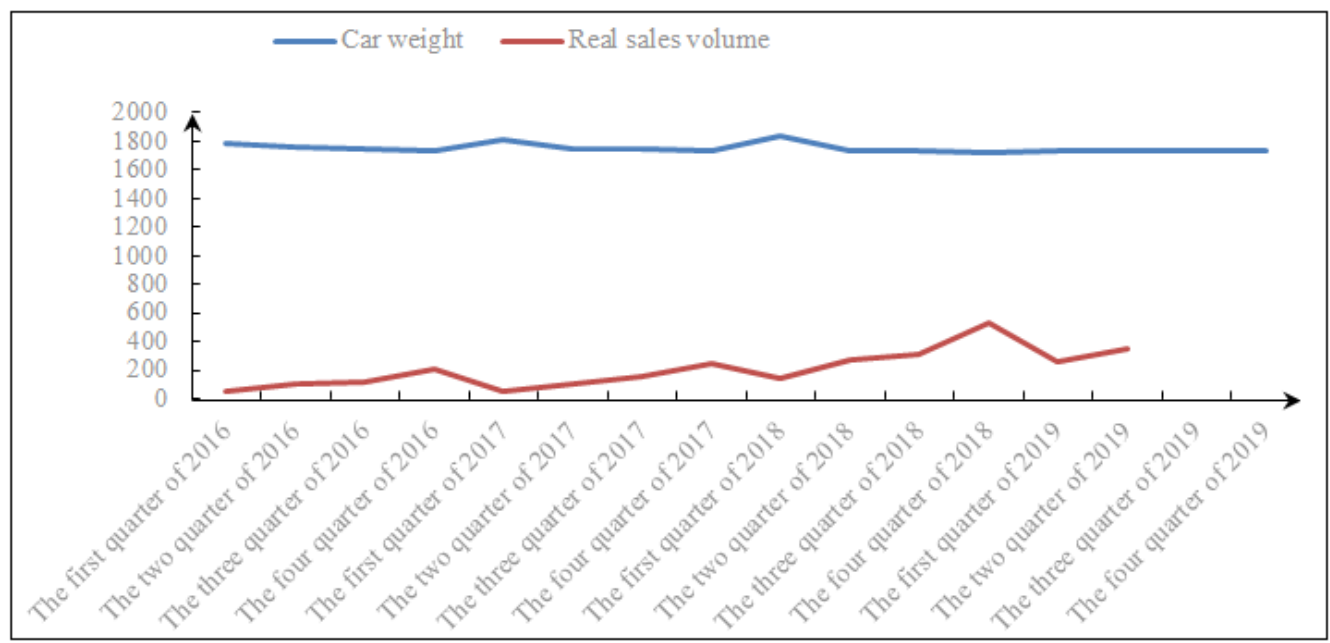

Fig-2: A broken line chart of the relationship between car weight and sales

By analyzing Table-1 and Fig-2, we find that there is a negative correlation between new energy sales and horsepower, a significant positive correlation between new energy sales and service mileage, and a relatively obvious positive correlation with vehicle weight.

\section{BP neural network prediction of new energy vehicle sales in China}

BP Network is a multilayer feedforward neural network. Among them, the training algorithm adjusting network weights is called BP learning algorithm. According to statistics, about $80 \%$ of neural network models [5] adopted BP network or its variant form. 
According to the principal component analysis method mentioned above, the three important performance indexes that affect the new energy vehicles are horsepower, vehicle weight and range. Therefore, this paper selects the horsepower, vehicle weight and range of new energy vehicles in the third quarter and fourth quarter of 2019, With the help of Matlab software BP neural network method for two quarters of sales forecast.

\section{Algorithm Steps}

The algorithm steps of BP neural network are as follows [5]:

Step1: Network initialization: set the initial values of each weight $\omega_{j i}^{(l)}(0)$ and threshold of the network $\theta_{j}^{l}$, generally the random number of $[0,1]$, as well as the error function, and give the maximum learning number $M$ and the accuracy value.

Step2: Input sample and expected output: randomly select the training sample and its corresponding target output.

Step3: Calculate the input of each layer $x^{(l)}=f\left(s^{(l)}\right)=f\left(W^{(l)} x^{(l-1)}\right)$;

\section{Step4: Calculation of training error;}

The training error of the input layer is $\delta_{j}^{(l)}=\left(d_{q i}-x_{j}^{(l)}\right) f^{\prime}\left(s_{j}^{(l)}\right)$,

The training error of hidden layer and input layer is $\delta^{(l)}=f^{\prime}\left(s_{j}{ }^{(l)}\right) \sum(n) k$;

Step5: Modified weight threshold;

$$
\begin{aligned}
& \omega_{j i}^{(l)}(k+1)=\omega_{j i}^{(l)}(k)+u \delta_{j}^{(l)} x_{i}^{(l-1)}+\eta\left(\omega_{j i}^{(l)}(k)-\omega_{j i}^{(l)}(k-1)\right), \\
& \theta_{j i}^{(l)}(k+1)=\theta_{j i}^{(l)}(k)+u \delta_{j}^{(l)}+\eta\left(\theta_{j i}{ }^{(l)}(k)-\theta_{j i}{ }^{(l)}(k-1)\right)
\end{aligned}
$$

Step6: Calculate global error. For errors between variables, see the following formula:

$$
E=\frac{\left(\sum_{q=1}^{Q}\left(E_{q}\right)^{2}\right)^{0.5}}{Q}
$$

Where $E_{q}=0.5 \sum_{j=1}^{n}\left(d_{q j}-y_{q j}\right)^{2}$, the error index of this paper is the mean square error.

Step7: Determine whether the network error meets the requirements: if the error meets the set requirements, that is, the network training terminates; otherwise, go to Step 2 and continue the next training cycle.

\section{The setting of the algorithm}

The number of nodes in the hidden layer is determined by the trial-compaction method, the formula is as follows:

$$
p=\sqrt{n+q}+\alpha \text {. }
$$

In the formula, $\mathrm{n}$ represents the number of input neuron nodes, q represents the number of output neuron nodes, and $\alpha$ is an integer in $[0,1]$.

In this paper, the input layer of BP neural network adopts purelin linear function transfer function, and tansig function is used as the transfer function of hidden layer. The LM algorithm is selected as the learning function of BP neural network prediction, and the linearization method and the steepest drop method are combined to find the optimal value quickly.

\section{Solution of the Model}

Using the horsepower, vehicle weight, mileage and sales indicators of new energy vehicles in each quarter of 2016-2019, forecast sales for the third and fourth quarters of 2019 missing from Table-1. The horsepower, vehicle weight and service mileage, which mainly affect the sales volume of new energy vehicles, are used as input data, and the sales volume of new energy vehicles is used as output data to train the data. Therefore, there are 14 nodes in the input layer and 1 node in the output layer. 
According to Table-1, the sales volume of new energy vehicles in the second half of 2019 is forecasted, and the BP neural prediction value is obtained and the error between the forecast and the real value is obtained. The specific results are shown in Table2.

Table-2: BP neural prediction results and errors

\begin{tabular}{|c|c|c|c|}
\hline Date & Real sales volume & BP neural prediction & Erros \\
\hline The first quarter of 2016 & 5.8 & 5.762 & 0.038 \\
\hline The second quarter of 2016 & 11.1 & 10.919 & 0.181 \\
\hline The third quarter of 2016 & 12.2 & 12.395 & -0.195 \\
\hline The fourth quarter of 2016 & 21.3 & 21.14 & 0.16 \\
\hline The first quarter of 2017 & 5.7 & 5.745 & -0.045 \\
\hline The second quarter of 2017 & 10.8 & 10.875 & -0.075 \\
\hline The third quarter of 2017 & 15.41 & 15.451 & -0.041 \\
\hline The fourth quarter of 2017 & 24.41 & 25.68 & -1.27 \\
\hline The first quarter of 2018 & 14 & 14.003 & -0.003 \\
\hline The second quarter of 2018 & 26.8 & 25.753 & 1.047 \\
\hline The third quarter of 2018 & 30.6 & 31.775 & -1.175 \\
\hline The fourth quarter of 2018 & 53.2 & 53.204 & -0.004 \\
\hline The first quarter of 2019 & 26.3 & 25.749 & 0.551 \\
\hline The second quarter of 2019 & 35.3 & 34.506 & 0.794 \\
\hline The third quarter of 2019 & & 25.469 & \\
\hline The fourth quarter of 2019 & & 27.904 & \\
\hline
\end{tabular}

Using the data in Table-2, an error graph is plotted (see Fig-3). As can be seen from Fig-3, the errors of prediction is about 0 , and only the difference between individual data is about 1. It has high prediction accuracy.

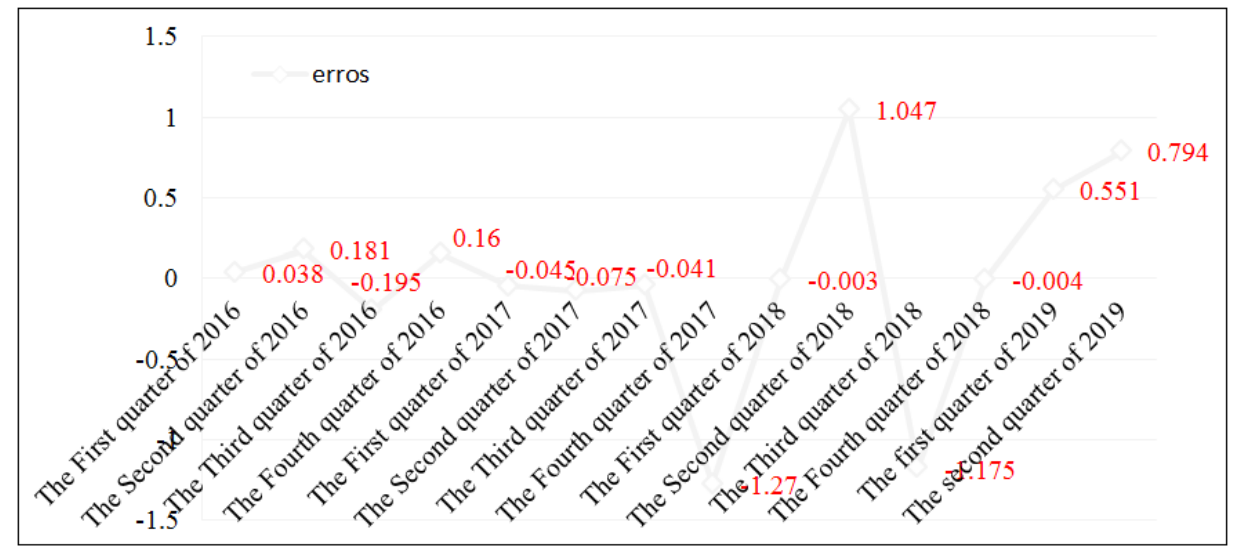

Fig-3: BP prediction value and real value error diagram

\section{CONCLUSIONS AND RECOMMENDATIONS}

Overall, from 2017 to 2019, the sales level of new energy vehicles showed an upward trend. With the introduction of some new energy policies, more and more consumers have gradually accepted new energy vehicles. Judging from our forecast sales in the third and fourth quarters of 2019 , sales of new energy vehicles showed solid growth. However, there are still many problems and areas for improvement in the process of development of new energy vehicles.

First of all, the market development of new energy vehicles is uneven. In a number of cities across the country to promote new energy vehicles, it is found that the sales of new energy vehicles account for a large proportion of the top five cities with economic development. Compared with the second, third and fourth tier cities, consumers in first-tier cities recognize new energy vehicles far more. Through consulting the data, it is found that there are four main reasons for this phenomenon: first, the subsidy policy of the first-tier cities is relatively favorable, and its licensing conditions are also relatively loose; second, the charging equipment of the firsttier cities is more adequate, and it has more perfect after-sales service; Third, the air pollution of second, third and fourth-tier cities is lower than that of first-tier cities, and the price of electric vehicles is much higher than that of fuel cars, coupled with insufficient subsidies, which makes it unacceptable to consumers. Fourth, the lack of pile charging equipment in the second, third and fourth line cities has had a great impact on the promotion of electric vehicles in these areas, which also shows that there is a serious market imbalance in the promotion process of new energy electric vehicles in China [6]. 
Secondly, the government should actively improve the policy of supporting the development of new energy. We will actively implement the preferential policies on finance and taxation for small and medium-sized enterprises and start-ups of new energy vehicles, and support the development of new energy automobile enterprises through policies such as tax concessions for high-tech enterprises and additional deductions for $R$ \& $D$ expenses. Encourage innovation and entrepreneurship related to the new energy automobile industry, encourage new energy vehicle companies to develop their own research and development to develop competitive products. At the same time, we should pay attention to create a good development environment for non-public new energy automobile enterprises. In terms of market access, examination and approval, treatment and service, we should provide loose and good services, mobilize the enthusiasm of private enterprises, and speed up the development of the new energy automobile industry [7]. There will be many other problems in the development of new energy vehicles, which need to be found and actively solved.

\section{REFERENCES}

1. Zheng, G. (2018). Interpretation and experience of made in China 2025, Manufacturing Technology and Machine Tools, 9, 7-8.

2. Ouyang, R. (2014). A comparative study based on automobile sales forecasting model. Jingdezhen Ceramic College.

3. Zhai, F., \& Lei, Y. (2019). Forecast model of sales volume of new energy vehicles in China. Journal of Henan Institute of Education, 28(1), 41-46.

4. Analysis on the present situation of sales in China's Automobile Industry in September 2019 National Automobile sales continue to decline. China Automobile Industry Association prospective Industry Research Institute collation.

5. Zhuo, J. (2011). Application of MATLAB in mathematical modeling, Beijing: Beijing University of Aeronautics and Astronautics Press.

6. Fan, G. (2019). An analysis of the present situation, problems and countermeasures of the development of new energy vehicles in China, Time Motor, (3),82-83.

7. Dong, T. (2019). Analysis and suggestion on the development of new energy automobile industry in China. Guangxi Quality Supervision Guide, 219(3), 101-102. 